\title{
Researches on Intelligent Parking Method of Parking Lot Based on Forecast and Multi-attribute Decision-making
}

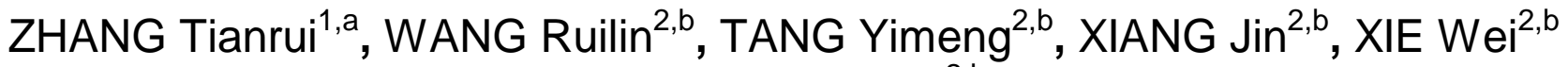 \\ and XU Shanshan ${ }^{2, b}$ \\ ${ }^{1}$ Full address of first author, including country \\ ${ }^{2}$ Full address of second author, including country \\ ${ }^{1}$ School of Mechanical Engineering, Shenyang University, Shenyang, China \\ ${ }^{2}$ School of International Studies, Shenyang University, Shenyang, China \\ atianjiangruixue@126.com, ${ }^{b}$ 332828953@qq.com
}

\begin{abstract}
Keywords: Prediction, Multiple Attribute Decision Making, Improved BP Neural Network, Optimal Routing

Abstract. For solving supply and demand balance between the parking space of parking lots and the car of the drivers, on the basis of analyzing time series forecasting techniques, the forecasting method using BP neural network algorithm was presented to forecast the parking lots free parking spaces, which was effective through MATLAB simulation. By analysis the drivers' main consideration about how to choose a parking space, the decision attribute matrix was identified by driving distance which was deduced through Dijkstra algorithm, walking distance was deduced through Euclidean distance and parking space environment value that was deduced through the entropy of triangular fuzzy number. Finally, using the grey correlation entropy method of MADM to sorting the effective free parking spaces, the optimal attributes of free parking spaces was the optimal free parking spaces.
\end{abstract}

\section{Introduction}

At present, the parking information that the city parking lot management system can provide was very limited, and it did not have the parking position forecast function ${ }^{[1-3]}$. In addition, the parking lot lacks a reasonable guiding mechanism. The situation of repeatedly looking for parking spaces had frequently occurred, and it was difficult to find the best parking position ${ }^{[4-6]}$. Solving this problem could alleviate the city traffic congestion, reducing vehicle exhaust emissions and noise pollution, improving the utilization of parking spaces and the economic benefits of parking ${ }^{[7-9]}$, it will also help to develop scientific traffic rules and regulations. Therefore, this paper has some theoretical and practical significance. Some researchers were combined with the Elman nerve network, conducting a short time prediction for the berth. And some research also studied the berth induction strategy inside the parking lot for the driver to shorten the time to find parking space ${ }^{[8-10]}$.

\section{Main influencing factors of intelligent parking}

According to the principle of combination of intelligent parking lot that people, car and parking lot should be combined in order to make the driver find an optimal berth, at least considering the driving distance, walking distance, parking environment and other influencing factors.

Determination of travel distance. In this paper, Dijkstra algorithm was used as the shortest path algorithm in the parking lot. Let the n-order directed weight graph $G(V, E)$, its point set $V=\left\{v_{1}, v_{2}, \ldots\right.$, $\left.v_{\mathrm{n}}\right\}$, edge set $E=\left\{e_{1}, e_{2}, \ldots, e_{\mathrm{n}}\right\}$, suppose the shortest distance between any two arbitrary related vertex $v_{i}$ and $v_{j}$ was $d_{i j}$, where $i, j=1,2, \ldots, n$, then the shortest distance matrix formula is:

$$
d_{i j}=\left\{\begin{array}{l}
d_{i j}, i \neq j \\
0, i=j
\end{array}\right.
$$

Determination of Walking Distance. Due to the subjective initiative of the person, the distance traveled by the driver after leaving the parking lot was a fuzzy value, but the shortest distance route 
was generally chosen. Therefore, this article applies Euclidean distance to determine the walking distance.

Determination of the Environmental information Value of the Berth. The berth environment contains multiple attributes, and each one was fuzzy, so the membership of the environmental attribute was determined by the triangular fuzzy number.

\section{Model establishment}

This paper systematically analyzes the present situation of berth forecasting and parking induction in domestic and international intelligent parking lot. Through the improvement of BP (Back Propagation) neural network, the berth information of intelligent parking lot was effectively predicted. The fuzzy multi-attribute decision-making method was applied to locate the best parking berth.

Time series prediction. Time series refers to a set of sequences which originated from a kind of phenomenon or event shown by the statistical indicators of various values at different time points or time on the in the order of time. According to the characteristics of the time series, it can be divided into time series of absolute number, time series of relative number and average time series. Among the time series of absolute number, they then can be divided into the time sequence and time-point sequence.

After the forecast was completed, a set of forecast criteria was needed to evaluate the results to determine the effectiveness of such a prediction method for the prediction of the problem. According to the characteristics of the problem, this paper will adopt the equal coefficient evaluation criteria to evaluate the accuracy of the forecast. $Y_{p}(t)$ represent the prediction value of the time sequence, and $Y_{r}(t)$ represents the real value of the time series, and the equal coefficient was as shown in equation (2). The higher the value, the higher the accuracy, the value range was $(0,1)$.

$$
E C=1-\frac{\sqrt{\sum\left(Y_{p}(t)-Y_{r}(t)\right)^{2}}}{\sqrt{\sum\left(Y_{p}(t)\right)^{2}}+\sqrt{\sum\left(Y_{r}(t)\right)^{2}}}
$$

BP neural network. BP neural network was a multi-layer feed-forward network trained by error reverse propagation, which was one of the most widely used neural network models. The neuron model was shown in Figure 1, the model structure was shown in Figure 2.

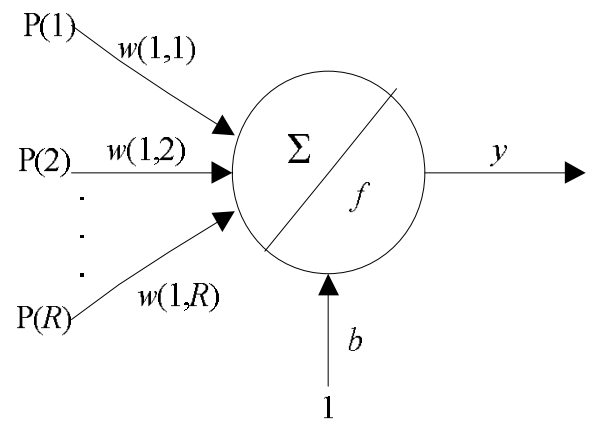

Fig. 1 Model of neuron

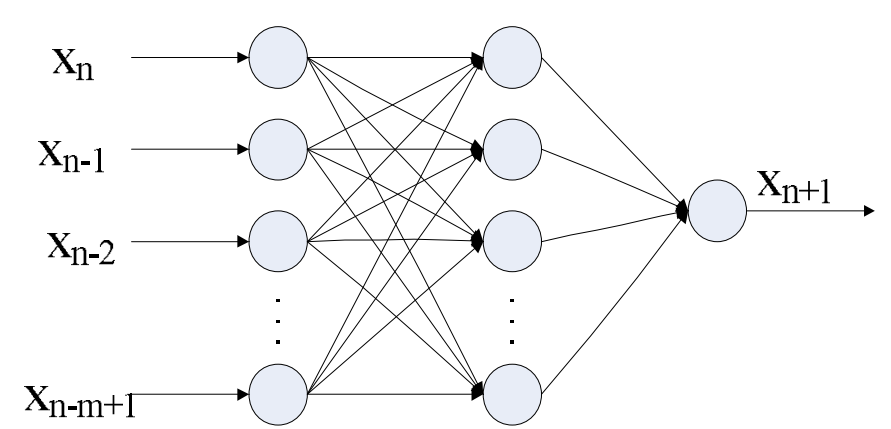

Fig. 2 Model structure of BP neural network

Assuming that the $n+1$ time series samples of a certain state characteristic parameter were $x(0)$, $x(1), \ldots, x(n)$, the value $x(n+1)$ at the time $n+1$ was predicted by the previous observation value $n$.

\section{Result analysis}

Choose an underground parking lot of a commercial square in Shenyang as an example to verify and analyze the case.

Prediction of excess berths. (1) Sample data selection. Select the sample data first before selecting the number of empty berths. As were shown in Tab. 2. 


\begin{tabular}{cccccc}
\hline Time period & $15: 00-16: 00$ & $16: 00-17: 00$ & $17: 00-18: 00$ & $18: 00-19: 00$ & $19: 00-20: 00$ \\
\hline $00-10$ & 192 & 201 & 206 & 182 & 175 \\
$10-20$ & 195 & 205 & 204 & 179 & 182 \\
$20-30$ & 194 & 205 & 204 & 178 & 183 \\
$30-40$ & 193 & 203 & 203 & 176 & 185 \\
$40-50$ & 193 & 203 & 99 & 175 & 179 \\
$50-60$ & 195 & 202 & 195 & 173 & 182 \\
\hline
\end{tabular}

(2) Data preprocessing. In order to improve the data processing speed, the free berth data was normalized by the formula (10) so that the empty berth data was mapped to the interval $[0,1]$. The preprocessing results were shown in Tab.2.

$$
x_{i}=\frac{x_{i}-x_{\min }}{x_{\max }-x_{\min }}
$$

Tab. 2 shows the data after normalization of Table 1

\begin{tabular}{cccccc}
\hline Time period & $15: 00-16: 00$ & $16: 00-17: 00$ & $17: 00-18: 00$ & $18: 00-19: 00$ & $19: 00-20: 00$ \\
\hline $00-10$ & 0.5758 & 0.8485 & 1.0000 & 0.2727 & 0.0606 \\
$10-20$ & 0.6667 & 0.9697 & 0.9394 & 0.1818 & 0.27274 \\
$20-30$ & 0.6364 & 0.9697 & 0.9394 & 0.1515 & 0.3030 \\
$30-40$ & 0.6061 & 0.9091 & 0.9091 & 0.0909 & 0.3636 \\
$40-50$ & 0.6061 & 0.9091 & 0.7879 & 0.0606 & 0.1818 \\
$50-60$ & 0.6667 & 0.8788 & 0.6667 & 0.0000 & 0.2727 \\
\hline
\end{tabular}

After the BP neural network training output results, to restore empty berth data, it was necessary to restore the normalized data. The reduction formula after normalization is:

$$
X_{i}=X_{i} \times\left(X_{\max }-X_{\min }\right)+X_{\text {min }}
$$

The network structure of the BP neural network was determined by the formula $n=\log _{2} N$, and the number of hidden neurons was calculated to be 5. And the Log-Sigmoid function $f(x)=1 /\left(1+e^{-x}\right)$ was chosen as the implicit layer transfer function of BP neural network.

(3) MATLAB simulation and results analysis. The number of training sessions was 1000 and the training accuracy was set to be 0.001 .

The figure was the actual line chart of the number of empty parking chart of the parking lot. And the figure was the predicting line chart of the number of empty berths for the standard BP neural network forecast line chart. The $E C$ value of the mean BP neural network was 0.9124 , and the predicted results of $E C$ value was 0.9798 . Therefore, the prediction model established in this paper shows certain accuracy.

Optimal berth decision analysis. There was a total number of eight parking areas, four elevator entrances and exits, one parking lot entrance and one parking lot exit in this parking lot. The Dijkstra algorithm calculates the minimum driving distance of the five berths $A_{1}-A_{5}$ to be $27,42,58,78$ respectively, and the environmental information values were shown in Tab.3.

Tab.3 Environmental information values of empty berths

\begin{tabular}{ccc}
\hline Parking area & Triangular fuzzy number & Expected value \\
\hline$A_{1}$ & $(0.050 .100 .15)$ & 0.1 \\
$A_{2}$ & $(0.450 .500 .55)$ & 0.5 \\
$A_{3}$ & $(0.550 .600 .65)$ & 0.6 \\
$A_{4}$ & $(0.350 .400 .45)$ & 0.4 \\
$A_{5}$ & $(0.250 .300 .35)$ & 0.3 \\
\hline
\end{tabular}

The best berth was chosen according to the decision steps described above.

(1) Determine the decision evaluation matrix: $R^{\prime}=\left[\begin{array}{ccc}27 & 18 & 0.1 \\ 42 & 5 & 0.5 \\ 58 & 6 & 0.6 \\ 72 & 10 & 0.4 \\ 78 & 17 & 0.3\end{array}\right]$ the corresponding berths of each

row were $A_{1}, A_{2}, A_{3}, A_{4}, A_{5}$; 
(2) Dimensionless calculation: $R=\left[\begin{array}{ccc}1 & 0 & 0 \\ 0.7059 & 1 & 0.8 \\ 0.3922 & 0.9231 & 1 \\ 0.1176 & 0.6154 & 0.6 \\ 0 & 0.0769 & 0.4\end{array}\right]$;

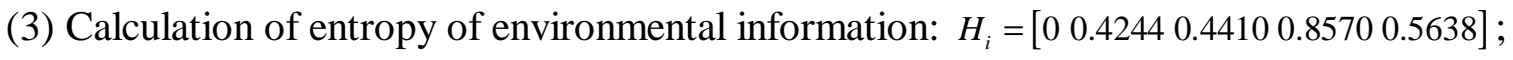

(4) Equilibrium degree calculation: $B_{i}=\left[\begin{array}{lllll}0 & 0.4952 & 0.5154 & 1 & 0.6579\end{array}\right]$;

(5) Entropy weight attribute matrix calculation: $V=\left[\begin{array}{ccc}0 & 0 & 0 \\ 0.2996 & 0.4244 & 0.3395 \\ 0.1729 & 0.4071 & 0.4410 \\ 0.1008 & 0.5274 & 0.5142 \\ 0 & 0.0434 & 0.2255\end{array}\right]$;

(6) Ideal program set for $e^{*}=\left[\begin{array}{lll}0 & 0 & 0.5142\end{array}\right]$;

(7) Gray correlation degree $R_{o i}=[0.77970 .48430 .59320 .68560 .7787]$ is determined by the degree of

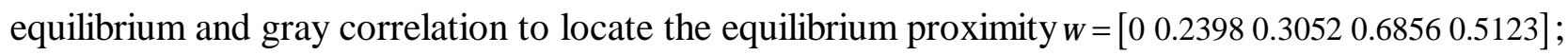

(8) The maximum value of equilibrium approximation was 0.6856 and the corresponding parking space was $A_{4}$. Therefore, $A_{4}$ was the best option of berth. It can be seen that the Dijkstra algorithm was very appropriate for the problems studied in this paper.

\section{Conclusions}

Aiming at solving the problem of parking nowadays, the method has introduced BP momentum and changeable learning rate method on the basis of standard BP neural network and brought about further improvement. The high prediction accuracy and validity for berth prediction possessed by the improved BP neural network has been shown by the results of MATLAB simulation. In addition, the optimal berth decision model was established by integrating the influencing factors like driving distance, walking distance, berth environment information and so forth. And the feasibility and efficiency of the model were verified through examples.

\section{Acknowledgements}

This work was financially supported by the Liaoning Dr. Research Start-up Foundation (20150192).

\section{References}

[1] J. Liu: A master's degree thesis of Hunan University. (2011)

[2] H. Li: A master's degree thesis of Xihua University. (2014)

[3] Z.Y. Li, F. Zong and B.H. Zhang: Infor. Sci. Vol. 29(2011), p. 1896

[4] F. Farivar, V. Majid and R. Omid: Energy Conversion and Management, Vol. 77(2014), p. 250

[5] Y.J. Ji, W. Wang and W. Deng: Jour. of Southeast University, Vol. 2(2009), p. 399

[6] X.Z. Zhang and Y.H. Qiu: Jour. of Wuhan University of Technology, Vol. 3(2011), p. 484

[7] Q. Chen, K.F. Yan, R.T. Wang, et al: Jour. of Tongji University, Vol. 5(2007), p. 607

[8] H.M. Cao and F. Han: International Conference on IMCCC, Vol. 9(2014), p. 393

[9] Y.Y. Yang: A master's degree thesis of Xi'an: Xidian University. (2014)

[10] S.X. Wang and A.Y. Li: Computer Science, Vol. 41(2014), p. 217 